\title{
Effect of chemical kinetic uncertainties on photochemical modeling results: Application to Saturn's atmosphere
}

\author{
M. Dobrijevic, J. L. Ollivier ^, F. Billebaud, J. Brillet, and J. P. Parisot \\ Observatoire de Bordeaux, BP 89, 32270 Floirac, France \\ Received 14 February 2002 / Accepted 21 October 2002

\begin{abstract}
Reaction rates included in photochemical models of planetary atmospheres carry with them a level of imprecision. This imprecision leads to an uncertainty in computed mole fractions of chemical species. We present estimations of the uncertainties on mole fraction profiles, obtained using a 1-D photochemical model applied to the study of hydrocarbon compounds in Saturn's atmosphere. We calculate the uncertainties as a function of altitude for each compound and derive the mean value and the standard deviation of the mole fraction. We show that the uncertainties on the mole fraction of hydrocarbons determined from a photochemical model are very significant. Ethane and acetylene, the most abundant hydrocarbons in Saturn's atmosphere after methane, are affected by an uncertainty factor of about 1.5, and the mole fraction of complex hydrocarbons, which are the products of a more complex chemistry, are affected by a large uncertainty factor which can reach 24 . We discuss the implications for the determination of the eddy diffusion coefficient. In particular, we show that uncertainties on the calculated
\end{abstract} \\ mole fractions produce a large uncertainty on the eddy diffusion coefficient profile in the lower atmosphere.
}

Key words. planets and satellites: individual: Saturn - methods: numerical

\section{Introduction}

Several authors have pointed out that imprecisions in reaction and photolysis rates used in photochemical models introduce significant uncertainties on computed concentrations. Early works on this topic were devoted to the Earth's atmosphere (Stolarski et al. 1978; Thompson \& Stewart 1991; Stewart \& Thompson 1996). More recently, Dobrijevic \& Parisot (1998) and Smith \& Raulin (1998) made similar studies of hydrocarbon photochemistry modeling in the atmospheres of Neptune and Titan, respectively. These studies were limited to 0-D box approximations (the vertical transport is not included in the continuity equation, see Sect. 2). Results showed that uncertainties in computed hydrocarbon concentrations are very significant. The authors quoted above suggested that uncertainties would be greater in 1-D models due to the possible propagation of uncertainties by vertical transport.

The atmospheres of the giant planets are dominated by molecular hydrogen and helium with a notable fraction of methane. Photochemical processes, subsequent to photolysis of methane and other compounds, lead to the production of a great variety of hydrocarbons. The knowledge of the altitude profiles of these minor compounds is of great interest to infer physical and chemical properties of the planets. In particular, comparisons between photochemical results and observations allow us to get some constraints on the eddy diffusion coefficient $(K(z))$,

Send offprint requests to: M. Dobrijevic,

e-mail: michel@observ.u-bordeaux.fr

* J.L. Ollivier works now in a private company. the tropospheric composition and the external flux of material. Since the uncertainties on the model parameters may lead to significant uncertainties on the computed mole fractions, it is of great importance to estimate these uncertainties in order to estimate the relevance of photochemical models to the determination of these atmospheric properties.

In the present paper, the photochemistry of hydrocarbon compounds in Saturn's atmosphere is studied, paying particular attention to imprecisions in photolysis and reaction rates and their effects on 1-D photochemical model results.

The 1-D photochemical model is briefly presented in Sect. 2. The Monte-Carlo technique used to generate reaction and photolysis rates randomly is presented in Sect. 3. Section 4 is devoted to the presentation of the results and finally, in Sect. 5 we discuss the implications of this work.

\section{Photochemical model}

The photochemical model used in the present study is based on the works of Dobrijevic (1996), Ollivier et al. (2000) and Ollivier (2000), who applied this model to Neptune and Saturn. The reader should refer to these papers for a detailed presentation of the characteristics of the code. In short, the different processes included in the model are the following: ultraviolet radiative transfer (no multiple scattering), photodissociation, chemical reactions, eddy diffusion vertical transport, molecular diffusion vertical transport, condensation, external influx of material and downward flux of atomic hydrogen. 
Determination of the abundances of the atmospheric compounds is obtained by solving the set of one dimensional continuity equations (one equation for each species, denoted by $i$ )

$$
\frac{\mathrm{d} n_{i}}{\mathrm{~d} t}=P_{i}-n_{i} L_{i}-\frac{\mathrm{d} \Phi_{i}}{\mathrm{~d} z}
$$

where $z$ is the altitude relative to the reference level (the reference level, $0 \mathrm{~km}$, of the atmosphere of the giant planets is taken at the 1 bar level), $n_{i}$ the concentration of compound $i, P_{i}$ the chemical production, $L_{i}$ the chemical loss and $\Phi_{i}$ the vertical flux. We must emphasize that we do not use the steady-state approximation, which allows us to write $\frac{\mathrm{d} n_{i}}{\mathrm{~d} t}=0$, but we prefer to solve the time-dependent diffusion equation with the CrankNicholson numerical method.

\subsection{Ultraviolet radiative transfer}

The production of minor hydrocarbons in the atmosphere of the giant planets is initiated by the photodissociation of the methane $\left(\mathrm{CH}_{4}\right)$ present in the upper atmosphere. The determination of the photodissociation rates requires the preliminary knowledge of the solar UV flux at every level in the atmosphere. Two approaches are possible to calculate this flux. The first one assumes that absorption of photons is the only important process and in this case we only have to calculate the attenuation of the solar flux through the atmosphere. The second one is a more detailed procedure which takes absorption and multiple scaterring into account. Ollivier et al. (2000) compared the results of the two approaches and showed that the abundances of ethane and acetylene were sensitive to multiple scattering. Since the calculation of multiple scattering enhances drastically the computing time, we chose to ignore multiple scattering in the determination of the UV solar flux in the present study.

The UV flux at a given altitude is a function of the solar UV flux at the top of the atmosphere, which is also a function of the solar cycle. The Infrared Space Observatory (ISO) satellite observations led to numerous new results with regard to the composition of Saturn's atmosphere. These observations were conducted in 1996, during the solar minimum. Therefore we chose a solar UV flux representative of solar minimum conditions to compare our results with ISO observations. Chemical lifetimes of major compounds are much larger than the period of Saturn's rotation. So, we used mean illumination conditions representative of 1996 (solar elevation and Sun/planet distance). All the results presented in this paper are obtained at the equator.

\subsection{Photolysis and reaction rates}

A major problem of the hydrocarbon photochemistry in planetary atmospheres is the lack of knowledge of the photodissociation process of methane, especially at Lyman- $\alpha$ wavelengths. Interpretation of methane photodissociation data has led to several schemes describing methane photolysis (see Smith \& Raulin 1998; Wilson \& Atreya 2000) and some of them are more or less favoured, but no conclusive experimental determination exists. Wilson \& Atreya (2000) compared the influence of the choice of the methane photolysis scheme on the abundance of hydrocarbons in the atmosphere of Titan, and they showed that it does not influence significantly the abundance of $\mathrm{C}_{2}$ compounds. We used in the present work the same scheme as in Ollivier et al. (2000) which comes from Mordaunt et al. (1993). Detailed work has been conducted by Moses et al. (2000) to determine the sensitivity of $\mathrm{CH}_{4}$ branching ratio on the hydrocarbon abundances in the atmosphere of Saturn. They found that the higher order hydrocarbons are particularly sensitive. This is a good example of the influence of uncertainties on photolysis rates on model results. Dobrijevic \& Parisot (1998) discussed the different sources of errors in the computations of the photolysis rates. Their conclusion was that the evaluation of uncertainties on these rates is very difficult to obtain and needs considerable study.

The photochemical scheme of hydrocarbons is an improved version of the one used by Ollivier et al. (2000). The chemical scheme contains 41 photodissociation processes and 170 reactions. The main correction of the reaction rates concerns the reaction:

$\mathrm{C}_{2} \mathrm{H}_{2}+\mathrm{C}_{2} \mathrm{H}_{6} \rightarrow \mathrm{C}_{2} \mathrm{H}_{3}+\mathrm{C}_{2} \mathrm{H}_{5}(\mathrm{R} 1)$.

Ollivier et al. (2000) included this reaction with a rate given by Laufer et al. (1983). The rate quoted by Laufer et al. (1983) concerns the reaction between $\mathrm{CCH}_{2}$ (triplet vinylidene) and $\mathrm{C}_{2} \mathrm{H}_{6}$. The removal of this reaction leads to a very different ethane abundance profile which is now in good agreement with the different observations of ethane in the stratosphere of Saturn. The effect of this correction on the mole fraction of the other major compounds in the atmosphere of Saturn is negligible.

The recent detection of the methyl radical $\mathrm{CH}_{3}$ in the stratosphere of Saturn by ISO reported by Bézard et al. (1998) have led to various discussions about its chemistry (Atreya et al. 1999; Ollivier et al. 2000; Moses et al. 2000). Bézard et al. (1998) pointed out that photochemical models were unable to reproduce the measured abundance, overestimating its column density. Moses et al. (2000) reconcile the observed abundance by choosing an appropriate rate coefficient (coming from MacPherson et al. 1983) for methyl recombination through a 3 -body reaction. This high rate coefficient leads to a higher loss rate of $\mathrm{CH}_{3}$ and therefore to a lower column abundance, in agreement with the result of Bézard et al. (1998). The methyl recombination rate is only known at room temperature and the problem arises from its extrapolation at low temperatures which may lead to very different values. Ollivier et al. (2000) used another expression that comes from Baulch et al. (1992) and it is the one we used here. Since the aim of the present work is to evaluate the magnitude of the uncertainties on hydrocarbon abundances, we will evaluate the uncertainty on methyl abundance, as well, and discuss the necessity to include a different reaction rate for methyl recombination.

\subsection{Saturn: Background atmosphere, thermal profile and eddy diffusion coefficient profile}

We use the same thermal profile as in Ollivier et al. (2000) (Fig. 1). It is constructed from three different observations, and 

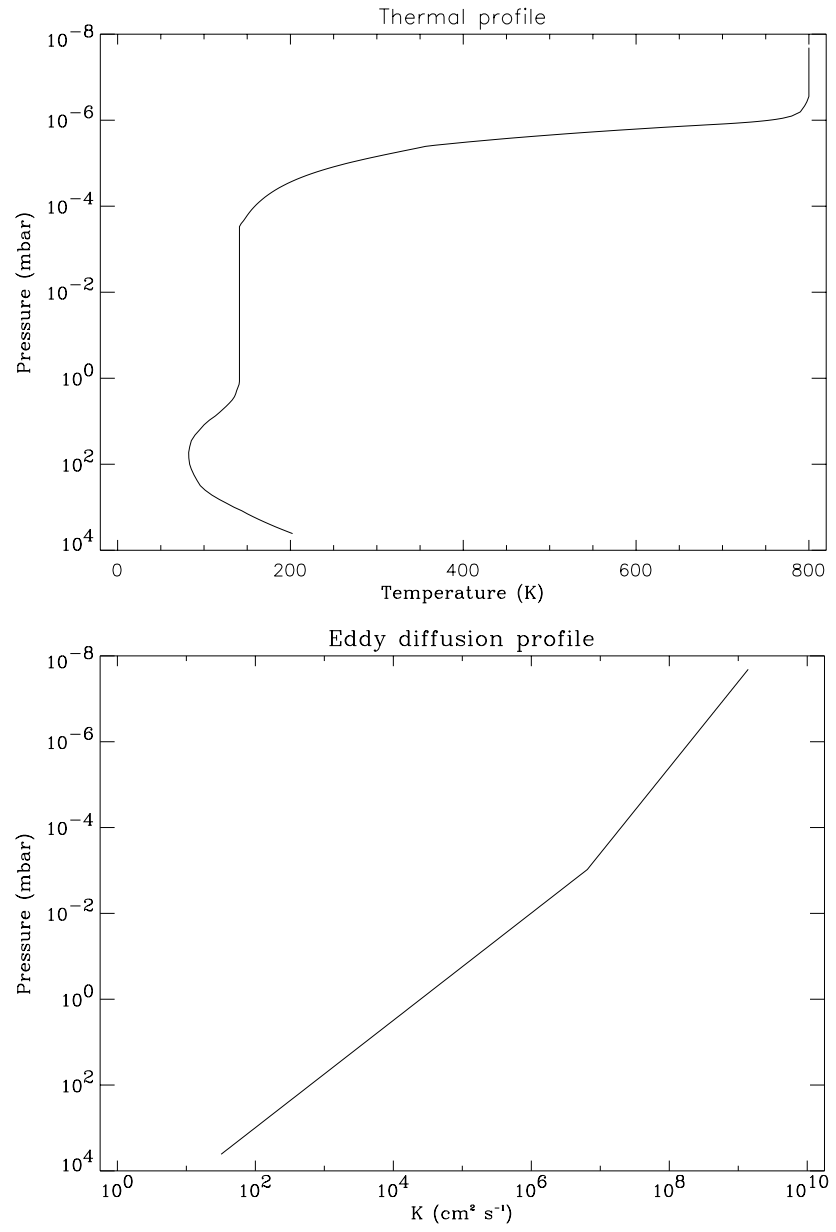

Fig. 1. Up: the thermal profile of Saturn's atmosphere. This profile is the same as in Ollivier et al. (2000). It is constructed from a set of observations probing the different levels of the atmosphere. Down: the nominal profile of the eddy diffusion coefficient.

the lower part is an evaluation assuming an adiabatic lapse rate. The model atmosphere extends from -100 to $3000 \mathrm{~km}$, and the reference level, $0 \mathrm{~km}$, is located at $1 \mathrm{bar}$. The background atmosphere is composed of molecular hydrogen and helium, with the respective mole fractions of 0.963 and 0.031 (Conrath et al. 1984). These values, deduced from analysis of Voyager infrared spectrometer (IRIS) spectra, have been re-evaluated by Conrath \& Gautier (2000), who found a greater He mole fraction (the helium mass fraction in Saturn's atmosphere should be $Y=0.18-0.25)$. Nevertheless, the effect of this larger helium abundance on hydrocarbon abundances should be small, and the evaluation of the abundance uncertainties is independent of the value of helium mole fraction. Methane molecules move from the deep atmosphere to the tropospheric levels, and are then transported into the stratosphere by eddy diffusion transport processes. The methane tropospheric mole fraction is set to $4.32 \times 10^{-3}$, in agreement with Courtin et al. (1984). As we only deal with carbon compounds, the abundances of the other tropospheric compounds, such as ammonia or phosphine, are set to 0 .

The eddy diffusion coefficient of Saturn's atmosphere has been improved since Ollivier et al. (2000). The removal of

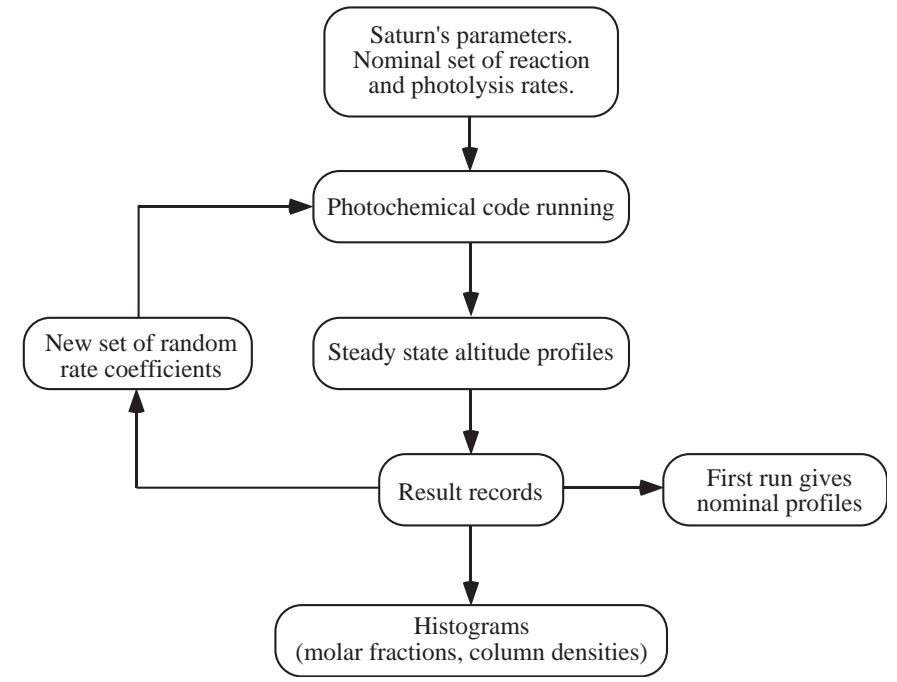

Fig. 2. Schematic presentation of the Monte-Carlo method used to study the effect of imprecisions in reaction rates on results of photochemical models.

reaction (R1) has modified the vertical distribution of abundance of ethane and we had to change the eddy diffusion coefficient to obtain better agreement with the ISO observations. The new eddy diffusion coefficient in Saturn's atmosphere is now

$$
\begin{aligned}
& K_{u}(z)=10^{8}\left(\frac{4.0 \times 10^{-6}}{P(z)}\right)^{\gamma_{1}} \quad P(z)<10^{-3} \mathrm{mbar} \\
& K_{l}(z)=K_{1}(z)\left(\frac{10^{-3}}{P(z)}\right)^{\gamma_{2}} \quad P(z)>10^{-3} \mathrm{mbar}
\end{aligned}
$$

where $K_{u}(z)$ and $K_{l}(z)$ are the eddy diffusion coefficient, respectively, for pressure lower and greater than $10^{-3} \mathrm{mbar}, P(z)$ is the pressure at the altitude $z, K_{1}(z)$ is given by $K_{u}(z)$ at the altitude where $P(z)$ is equal to $10^{-3}$ mbar, $\gamma_{1}=0.5$ and $\gamma_{2}=0.8$.

Atomic hydrogen is produced in the upper part of the atmosphere from the photodissociation of molecular hydrogen by energetic ultraviolet photons. The diffusion of these atoms in the lower part of the atmosphere is similar to a downward flux of molecules or atoms and we take this influx of atomic hydrogen into account through a flux of $10^{8} \mathrm{H}$ atoms $\mathrm{cm}^{-2} \mathrm{~s}^{-1}$.

\section{Monte-Carlo simulation of reaction rate uncertainties}

The determination of the uncertainties on the mole fractions follows the technique used by Stolarski et al. (1978). First, we calculate with a 1-D photochemical model the nominal abundances using nominal reaction rates. Then, we calculate a random rate for each reaction and the calculations are performed for each of these sets of perturbed rates (see Fig. 2). We chose to perform 2000 runs in order to have a large sample of results and to determine a statistical estimate of the mean mole fractions and standard deviations.

The assignment of the errors on reaction rates is based on the work of Baulch et al. (1992). A major fraction of our reaction rates comes from this reference which also gives an estimation of the errors. Let us denote $\log \left(k_{0 i}\right)$ the logarithm of 
the initial value of the $i$ th rate coefficient. The set of $k_{0 i}$ is the values used for the first run of the photochemical code, which we call "nominal run". The probability distribution followed by the perturbed reaction rates is the lognormal distribution (Stewart \& Thompson 1996), whose probability density function is given by

$$
f(x)=\frac{1}{\sqrt{2 \pi} \sigma x} \exp \left(-\frac{(\ln (x)-\mu)^{2}}{2 \sigma^{2}}\right)
$$

where $x \in] 0, \infty[, \mu$ and $\sigma$ are the parameters of the distribution. When a random variable follows a lognormal distribution, the logarithm of this variable follows the normal distribution. Thus, the new value ("perturbed") $k_{i}$ of the reaction rate is given by

$\log \left(k_{i}\right)=\log \left(k_{0 i}\right)+\epsilon_{i} \log \left(F_{i}\right)$

where $\epsilon_{i}$ is a random number with a normal distribution of null mean and unity standard deviation and $F_{i}$ is the uncertainty factor of the $i$ th reaction (or photodissociation process). In order to dismiss extreme values of $k_{i}$ which can be obtained by Eq. (5), we reject values of $|\epsilon|$ greater than 2 . Uncertainty factors of reaction rates are evaluated by experimenters. A list of these factors, which are often greater than 2 and can reach 10, can be found in Dobrijevic \& Parisot (1998). Moreover, the reaction rates and their uncertainties are determined for a given temperature range which is often not representative of the giant planets' atmospheric temperatures $(<200 \mathrm{~K})$. The extrapolation at low temperatures is thus another source of uncertainty. Stewart \& Thompson (1996) have conducted a detailed study of the behavior of the uncertainties on reaction rates as a function of temperature, which allows them to estimate the magnitude of the uncertainty factor at a temperature different from the one used in the experiment. This study is based on the data given by rate compilations (DeMore et al. 1994; Atkinson et al. 1992) which give the Arrhenius coefficient and the activation temperature, and the associated errors, at a given temperature. It is then possible to calculate the propagation of the errors at different temperatures and especially at lower temperature. The compilation of Baulch et al. (1992) does not provide such information and we have to assume that the uncertainty factor is constant with the temperature. In their critical review, Baulch et al. (1992) indicate that the estimation of uncertainties on reaction rates is a difficult task and a lot of them might be underestimated, while some of them are not known. There are some studies that provide rates at low temperature which would be useful to reduce the amount of uncertainty in some reactions. For instance, Canosa et al. (1997) reported rates between 23 and $295 \mathrm{~K}$ of reactions of $\mathrm{CH}$ with several hydrocarbons. However, the products of these reactions are not known. It would be possible to form some hypothesis about these products but this will incorporate additional uncertainties. In addition, some measurements (Sims et al. 1993) show that the difference of rates measured at low temperature and rates obtained from extrapolation from measurements done at $300 \mathrm{~K}$ are reaction dependant. It is consequently difficult to estimate the uncertainty factors of these reactions at low temperature. In any case, these factors would be probably greater than the lower limit we use in the present study. Since almost all the
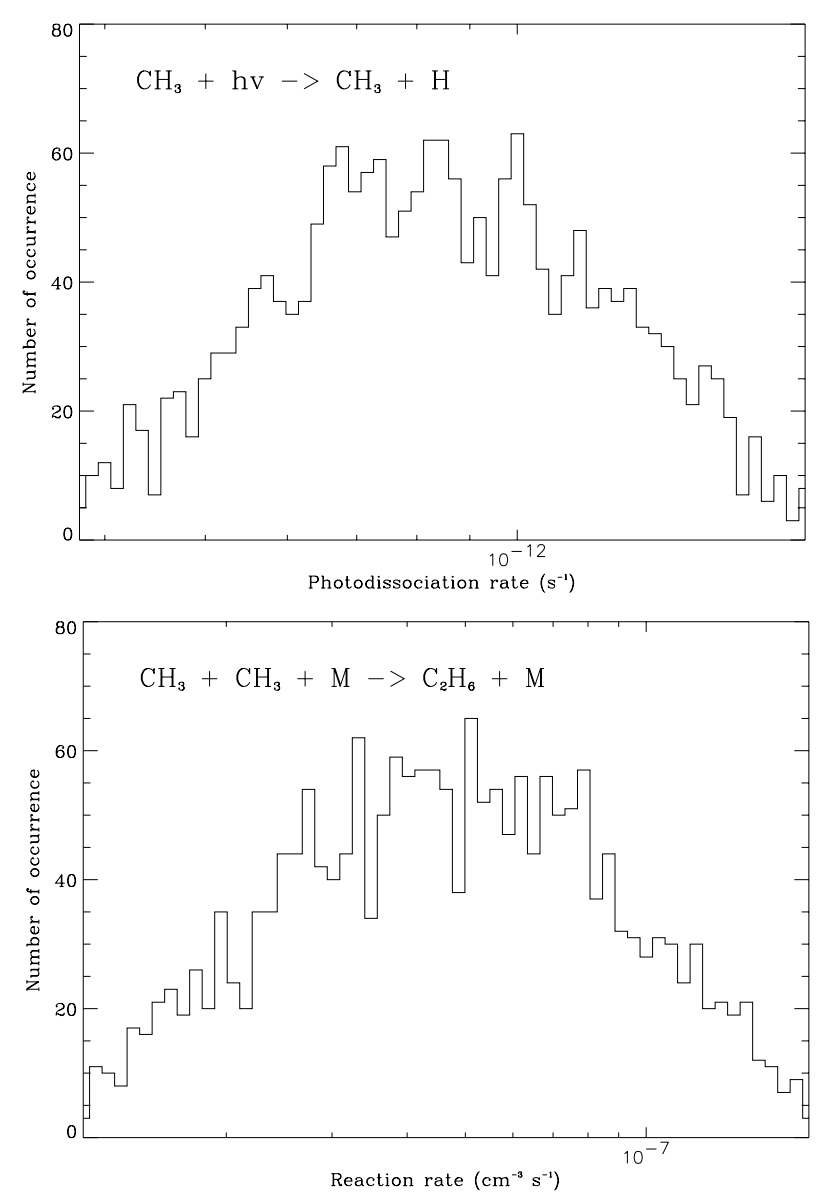

Fig. 3. $U p$ : histogram showing the distribution of the photodissociation rate of methane at $10^{-4}$ mbar, leading to the production of the methyl radical. The uncertainty factor is set to 1.5. Down: histogram showing the distribution of the recombination rate of methyl through three-body reaction, at 0.1 mbar. The uncertainty factor is set to 2 . The distributions have been obtained for 2000 runs.

uncertainty factors of the reaction rates we used in our model are not known or very imprecise in the temperature range of Saturn atmosphere, a lower limit of $F_{i}=2.0$ for each uncertainty factor is used at all pressure levels. The ter-molecular reaction uncertainties are treated in the same way as bimolecular reactions: we calculate the reaction rate from the high pressure and low pressure rates and we apply the same uncertainty factor $F_{i}=2.0$ to the result. This treatment of the uncertainty factor allows us to estimate a lower limit of the errors on the abundances of hydrocarbons in the atmosphere of Saturn determined from photochemical modeling.

Dobrijevic \& Parisot (1998) discussed the different sources of uncertainty in the photolysis rates. Taking branching ratio, solar flux and cross section uncertainties into account, they estimated that an uncertainty factor of 1.5 for $J(i, z)$ is a lower limit $(J(i, z)$ is the photolysis rate at altitude $z$ for the compound $i)$. We use this value in the present work.

Figure 3 presents the histograms of a photolysis process $\left(\mathrm{CH}_{4}+\mathrm{h} v \rightarrow \mathrm{CH}_{3}+\mathrm{H}\right)$ and a three-body reaction $\left(\mathrm{CH}_{3}+\mathrm{CH}_{3}+\right.$ $\left.\mathrm{M} \rightarrow \mathrm{C}_{2} \mathrm{H}_{6}+\mathrm{M}\right)$ as obtained after a simulation of 2000 runs at $670 \mathrm{~km}\left(10^{-4} \mathrm{mbar}\right)$ and $440 \mathrm{~km}(0.1 \mathrm{mbar})$ in the stratosphere of Saturn, respectively. 

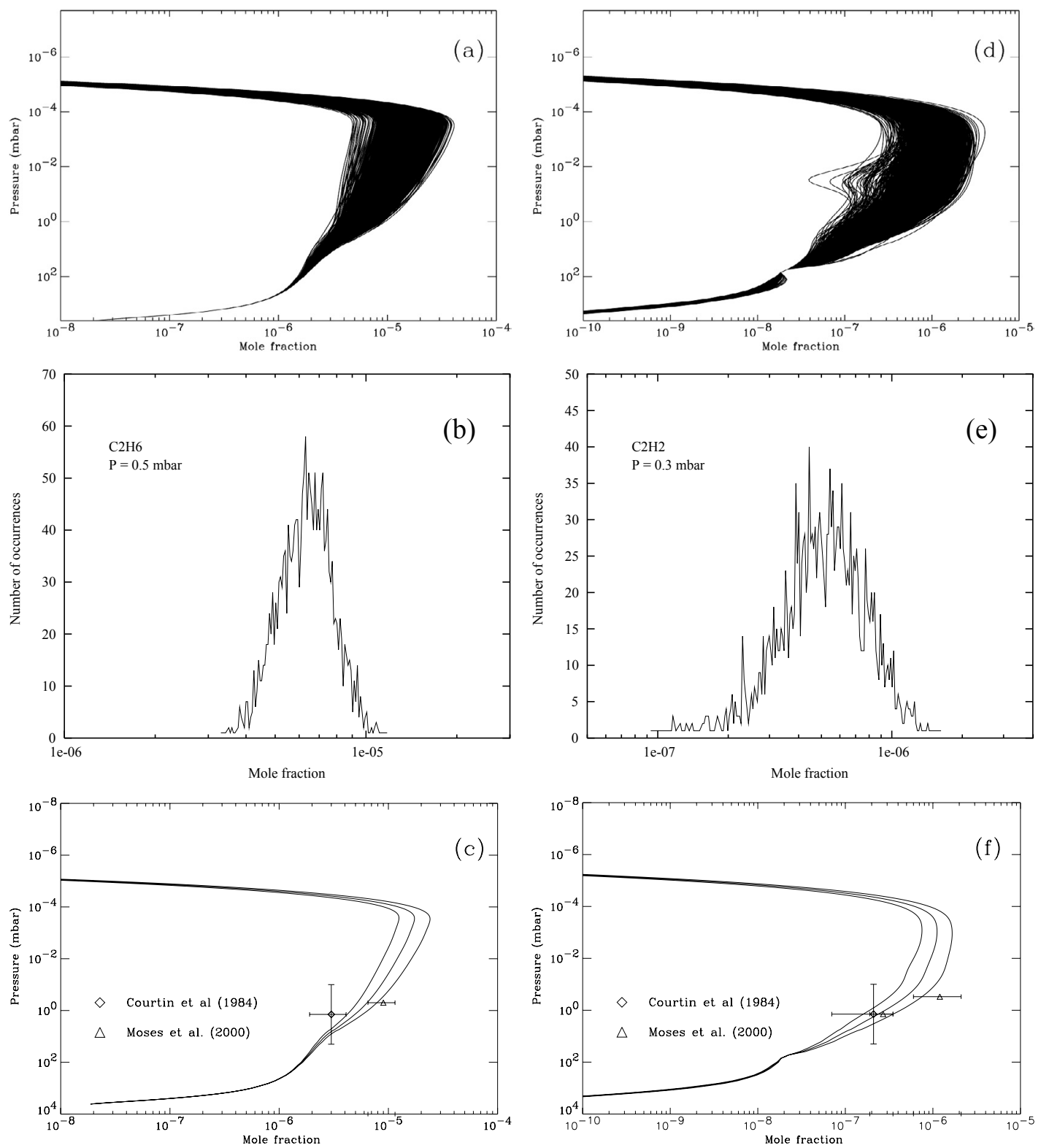

Fig. 4. Results of 2000 runs for ethane (left) and acetylene (right). The uncertainty factor was set to 1.5 for the photodissociation processes and to 2 for the two or three-body reactions. Two estimations of the ethane mole fraction, obtained from different observations, have also been indicated. The diamond corresponds to the results of Courtin et al. (1984), from Voyager observations, and the triangle, to the results of Moses et al. (2000), who retrieved this mole fraction from comparison between modeled spectra and ISO spectra. Top (a) and d)): 2000 mole fraction profiles as a function of pressure. Middle (b) and e)): distributions of the mole fractions at 0.5 mbar (left) and 0.3 mbar (right). Bottom (c) and f)): Mean (thick line) and standard deviation (thin line) of mole fractions as a function of pressure.

\section{Results: Uncertainties on hydrocarbon abundances}

Imprecision effects in rate coefficients on photochemical model results in the stratosphere of Saturn are now examined in detail. The 2000 runs allow us to make a determination of the statistics of the hydrocarbon abundance distributions. Figures $4 \mathrm{a}$ to $4 \mathrm{f}$ show the results for the two major products of methane photochemistry, ethane $\left(\mathrm{C}_{2} \mathrm{H}_{6}\right)$ and acetylene $\left(\mathrm{C}_{2} \mathrm{H}_{2}\right)$. In Figs. $4 \mathrm{a}$ and $4 \mathrm{~d} 2000$ profiles are drawn as a function of pressure level for ethane and acethylene, respectively. Figures $4 \mathrm{~b}$ and $4 \mathrm{e}$ show the distributions of mole fractions at two distinct pressure levels
(0.5 mbar and 0.3 mbar respectively). Mean and standard deviation as a function of pressure level are shown in Figs. 4c and $4 \mathrm{f}$. The mole fraction of $\mathrm{C}_{2} \mathrm{H}_{6}$ and $\mathrm{C}_{2} \mathrm{H}_{2}$ retrieved by Courtin et al. (1984) and Moses et al. (2000) are also indicated. For a detailed description of Saturn observations, see Moses et al. (2000).

Figures $4 \mathrm{a}$ and $4 \mathrm{~d}$ clearly show that the mole fraction profiles of ethane and acetylene are extremely affected by the change of reaction rates. From one run to another, the slope of the altitude profile can be very different. In particular, the maximum of the mole fraction may not peak at the same pressure level. 


\subsection{About the comparison with observations}

In order to make comparisons with observations, we decided to use the data provided by ISO spectra, because they consist of a coherent (i.e. from the same observing technique) set of mole fractions and column densities. Moses et al. (2000) derived column densities and mole fractions from these spectra, so we can compare our values with the ones they obtained. However, doing this, we must be aware that we are not performing a "direct" comparison with data, as the values obtained by Moses et al. (2000) were obtained themselves through comparisons with results of their photochemical model. Indeed, they ran their photochemical code and processed their results through a radiative transfer code which produced synthetic spectra that they compared with ISO spectra. Therefore, although we subsequently call them "ISO observations", their results are not "raw data" but model-dependent observing results (in the sense that the results depend on abundances retrieved from the photochemical models). In the ideal case, the correct way to make such an analysis would be to compute synthetic spectra with our 2000 photochemical model run results, and then compare them with ISO spectra. As a matter of fact, this represents an enormous amount of computing time, which we chose not to perform. However, forthcoming work will have to deal with the computation of synthetic spectra in order to make more valuable comparisons. Within the present work, we will make comparisons with Moses et al. (2000) results, bearing in mind the limitations of this procedure.

\subsection{Comparisons with the results of Moses et al. (2000)}

From Figs. 4a to $4 \mathrm{f}$, it is clear that the photochemical model used in the present work is in "perfect" agreement with the $\mathrm{C}_{2} \mathrm{H}_{6}$ and $\mathrm{C}_{2} \mathrm{H}_{2}$ observations in the sense that the uncertainties of computed profiles and observations overlap. Our results show that the logarithms of the mole fractions are, at each pressure level, normally distributed (see Figs. 4b and 4e). In particular, the distribution of the ethane mole fractions overlaps the abundance derived from ISO observations which is $9 \pm 2.5 \times 10^{-6}$ at $0.5 \mathrm{mbar}$ (Moses et al. 2000). Over the 2000 runs, 908 computed mole fractions are in agreement with this value. In the case of acetylene, 584 computed mole fractions are in agreement with the value also derived from ISO: $1.2_{-0.6}^{+0.9} \times 10^{-6}$ at 0.3 mbar.

Given $y_{0 i}(z)$, the mean mole fraction, and $F_{y i}(z)$, the uncertainty factor at altitude $z$ for compound $i$, the computed mole fraction is then equal to:

$\log \left(y_{i}(z)\right)=\log \left(y_{0 i}(z)\right) \pm \log \left(F_{y i}(z)\right)$.

The standard deviations of the distributions shown in Figs. $4 \mathrm{~b}$ and $4 \mathrm{e}$ are equal to the logarithm of the uncertainty factors. Table 1 summarizes the results for all hydrocarbons: it shows the maximum uncertainty factor for each compound and the corresponding pressure level. The lowest values of this factor are obtained for methane, ethane and acetylene whose maximum uncertainty factors are respectively of the order of 1.04, 1.4 and 1.6. For ethane, the maximum uncertainty factor is
Table 1. Maximum uncertainty factor $\left(F_{y}\right)$ and corresponding pressure level for the whole set of hydrocarbon compounds.

\begin{tabular}{ccc}
\hline \hline Compound & Maximum uncertainty factor & Pressure (mbar) \\
\hline $\mathrm{CH}_{3}$ & 2.801 & $0.24768 \times 10^{-1}$ \\
$\mathrm{CH}_{4}$ & 1.042 & $0.29270 \times 10^{-6}$ \\
$\mathrm{C}_{2} \mathrm{H}_{2}$ & 1.624 & $0.29156 \times 10^{-1}$ \\
$\mathrm{C}_{2} \mathrm{H}_{4}$ & 2.577 & $0.17862 \times 10^{-1}$ \\
$\mathrm{C}_{2} \mathrm{H}_{6}$ & 1.420 & $0.29270 \times 10^{-6}$ \\
$\mathrm{C}_{3} \mathrm{H}_{2}$ & 14.743 & $0.34310 \times 10^{-1}$ \\
$\mathrm{C}_{3} \mathrm{H}_{3}$ & 11.833 & $0.34310 \times 10^{-1}$ \\
$\mathrm{CH}_{2} \mathrm{CCH}_{2}$ & 10.577 & $0.29156 \times 10^{-1}$ \\
$\mathrm{CH}_{3} \mathrm{C}_{2} \mathrm{H}$ & 5.494 & $0.15154 \times 10^{-1}$ \\
$\mathrm{C}_{3} \mathrm{H}_{6}$ & 3.952 & $0.68074 \times 10^{-3}$ \\
$\mathrm{C}_{3} \mathrm{H}_{8}$ & 2.839 & $0.12846 \times 10^{-1}$ \\
$\mathrm{C}_{4} \mathrm{H}_{2}$ & 8.310 & $0.34310 \times 10^{-1}$ \\
$\mathrm{C}_{4} \mathrm{H}_{3}$ & 8.093 & $0.34310 \times 10^{-1}$ \\
$\mathrm{C}_{4} \mathrm{H}_{4}$ & 4.359 & $0.12076 \times 10^{+2}$ \\
$\mathrm{C}_{4} \mathrm{H}_{6}$ & 9.328 & $0.71638 \times 10^{+2}$ \\
$\mathrm{C}_{4} \mathrm{H}_{10}$ & 2.660 & $0.61030 \times 10^{-6}$ \\
$\mathrm{C}_{6} \mathrm{H}_{2}$ & 13.343 & $0.34310 \times 10^{-1}$ \\
$\mathrm{C}_{8} \mathrm{H}_{2}$ & 24.484 & $0.34310 \times 10^{-1}$ \\
$\mathrm{C}_{6} \mathrm{H}$ & 14.141 & $0.34310 \times 10^{-1}$ \\
${ }^{1} \mathrm{CH}_{2}$ & 2.336 & $0.29270 \times 10^{-6}$ \\
$\mathrm{C}_{3} \mathrm{H}_{7}$ & 3.156 & $0.41266 \times 10^{-2}$ \\
$\mathrm{C}_{4} \mathrm{H}$ & 8.900 & $0.34310 \times 10^{-1}$ \\
${ }^{3} \mathrm{CH}_{2}$ & 3.070 & $0.34310 \times 10^{-1}$ \\
$\mathrm{C}_{2}$ & 2.224 & $0.34310 \times 10^{-1}$ \\
$\mathrm{C}_{2} \mathrm{H}$ & 2.076 & $0.10500 \times 10^{-3}$ \\
$\mathrm{C}_{2} \mathrm{H}_{3}$ & 0.013 & $0.40362 \times 10^{-1}$ \\
$\mathrm{C}_{2} \mathrm{H}_{5}$ & 3.791 & $0.15154 \times 10^{-1}$ \\
$\mathrm{C}_{3} \mathrm{H}_{5}$ & $\mathrm{C}$ & \\
\hline & 12.774 & $0.47460 \times 10^{-1}$ \\
\hline & &
\end{tabular}

maximal at a very high level in the atmosphere and decreases towards the lower levels. The uncertainty factor is actually quite constant in the stratosphere (Fig. 4c) and lies between 1.2 and 1.37 for pressures between 1 mbar and $10^{-5}$ mbar. The maximum uncertainty factor for acetylene is reached at a pressure level of $3 \times 10^{-2}$ mbar. Between 1 mbar and $10^{-5}$ mbar, this factor lies between 1.46 and 1.62 .

The situation for the other hydrocarbons is very different: the maximum uncertainty factor is larger than 2 and can reach such an extreme value as 24 . Complex hydrocarbons with more than three carbon atoms are the results of many reactions or photodissociation and have thus the most imprecise abundances. The increase in the complexity of the production processes leads to an increase in the uncertainty on the abundance.

These results have sizeable implications. For instance, Ollivier et al. (2000) stated that the photochemical scheme leading to the production of species like methylacetylene $\left(\mathrm{CH}_{3} \mathrm{C}_{2} \mathrm{H}\right)$ or diacetylene $\left(\mathrm{C}_{4} \mathrm{H}_{2}\right)$ must be improved. Indeed, the computed mole fraction profile of these two compounds 
obtained with the nominal reaction rates showed a large disagreement with the observed estimation, retrieved from the ISO observations (de Graauw et al. 1997; Moses et al. 2000). Actually, if we take the uncertainty of the computed mole fraction of methylacetylene into account, we see that the value determined by Moses et al. (2000) is then in agreement with the model prediction (Fig. 5, left). Moses et al. (2000) obtained a $\mathrm{CH}_{3} \mathrm{C}_{2} \mathrm{H}$ mole fraction of $1.8 \pm 0.5 \times 10^{-9}$ at $0.5 \mathrm{mbar}$ and we find that, at this level, our computed mole fraction lies between $1.25 \times 10^{-10}$ and $1.7 \times 10^{-9}(1-\sigma$ limits $)$ at 0.5 mbar. The mole fraction of diacetylene $\mathrm{C}_{4} \mathrm{H}_{2}$ has also been determined from ISO observation: Moses et al. (2000) derived a value of $2.1 \pm 0.5 \times 10^{-10}$ at $0.5 \mathrm{mbar}$. At this level, we find that the $1-\sigma$ limits of $\mathrm{C}_{4} \mathrm{H}_{2}$ mole fraction are $3.1 \times 10^{-10}$ and $3.0 \times 10^{-9}$. The observed value is thus outside the $1-\sigma$ limit. Only 135 runs (7\%) give profiles of $\mathrm{C}_{4} \mathrm{H}_{2}$ that match observations at 0.5 mbar. However, it is important to note that we probably estimate in the present work a lower limit of the uncertainty on the modeled abundance profiles. The uncertainty factor on chemical reactions is equal to 2, which is lower than the uncertainty factor for a large number of reactions (Baulch et al. 1992; Dobrijevic $\&$ Parisot 1998). Consequently, considering the uncertainties on the reaction rates, the chemical scheme used by Ollivier et al. and by the present model is satisfactory.

Figure 6 presents the mean and the standard deviation of the allene $\left(\mathrm{CH}_{2} \mathrm{CCH}_{2}\right)$ mole fraction whose mole fraction uncertainty is one of the most significant. Ollivier et al. (2000) showed that the process leading to the production and destruction of this compound implied numerous reactions between hydrocarbons, and the complexity of the process thus increases the uncertainty on the abundance. The upper limit of the allene abundance has been estimated by Moses et al. (2000). This value is not drastically greater than the $1 \sigma$ upper limit that we have obtained. No conclusion can be drawn from this result regarding the chemistry nor the eddy diffusion coefficient.

The column density is another relevant quantity in order to compare observations and photochemical models. It gives no information on the dependence of the abundances on altitude, but it is suitable to constrain the chemistry or the eddy diffusion profile. Figure 7 presents the column density distributions of six hydrocarbons obtained from our model. In comparison, the column densities that have been determined from ISO observations by Moses et al. (2000) are also shown. Regarding methylacetylene, its modeled column density is in very good agreement with the observed value. Nevertheless, since the determination of a column density does not include information about the vertical distribution of a compound, which has a large influence on the outgoing infrared flux, this result has to be considered carefully and there is no firm evidence that our modeled $\mathrm{CH}_{3} \mathrm{C}_{2} \mathrm{H}$ profile could reproduce the ISO infrared spectra. The column density of $\mathrm{C}_{4} \mathrm{H}_{2}$ retrieved by Moses et al. (2000) lies at the lower limit of our distribution. Only a few sets of chemical rates give a column density of $\mathrm{C}_{4} \mathrm{H}_{2}$ in agreement with the ISO value. Moses et al. (2000) derived an upper limit for the $\mathrm{C}_{2} \mathrm{H}_{4}$, the $\mathrm{CH}_{2} \mathrm{CCH}_{2}$ and the $\mathrm{C}_{3} \mathrm{H}_{8}$ column density. The agreement with the modeled distribution is very good and only a minor fraction of the distribution is excluded by the observations. The most interesting case is obtained for the methyl
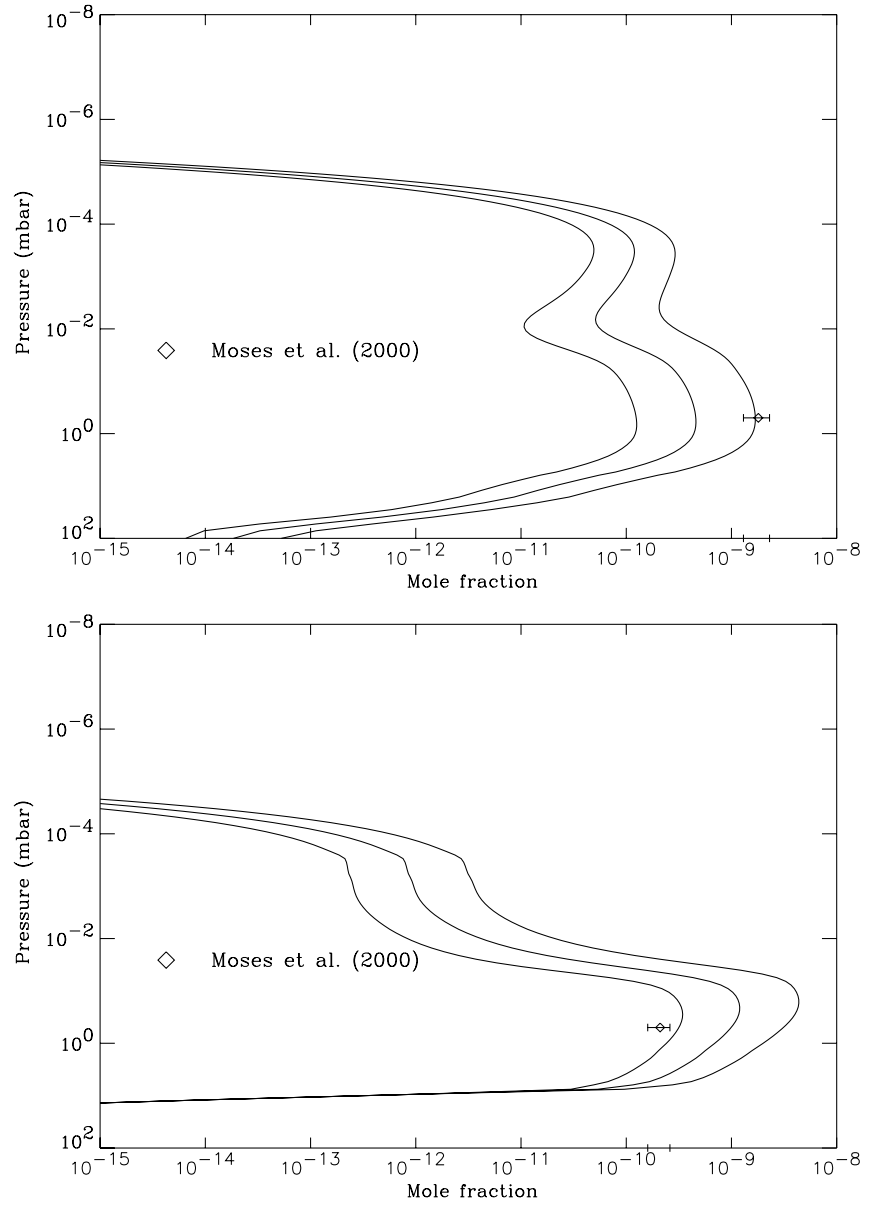

Fig. 5. Mean (thick line) and standard deviation (thin line) of (up) methylacetylene $\left(\mathrm{CH}_{3} \mathrm{C}_{2} \mathrm{H}\right)$ and (down) diacetylene $\left(\mathrm{C}_{4} \mathrm{H}_{2}\right)$ mole fraction. The diamonds show the value derived by Moses et al. (2000) on the methylacetylene and diacetylene mole fraction from the analysis of the ISO spectra.

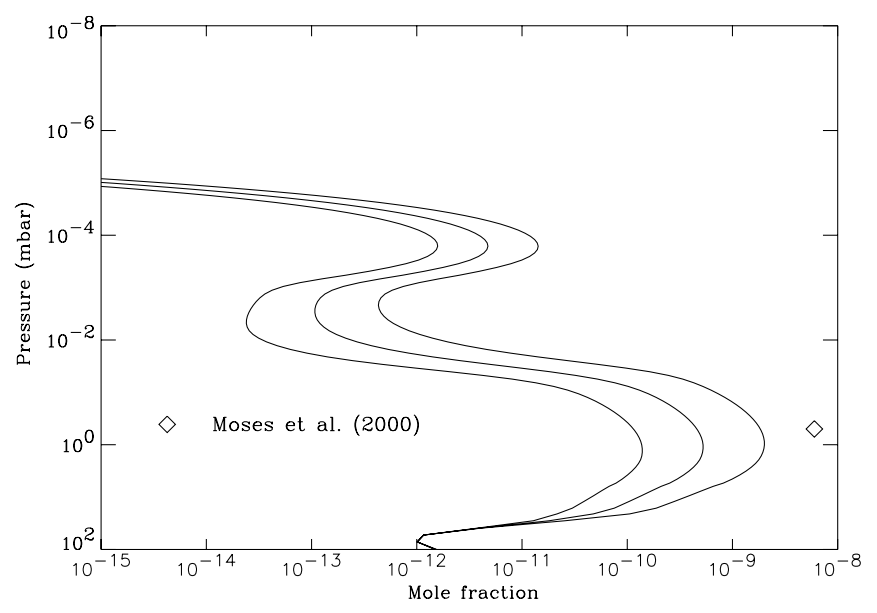

Fig. 6. Mean (thick line) and standard deviation (thin line) of the allene $\left(\mathrm{CH}_{2} \mathrm{CCH}_{2}\right)$ mole fraction. The diamond show the upper limit derived by Moses et al. (2000) on the allene mole fraction from the analysis of the ISO spectra.

radical whose column density has been determined through the analysis of ISO observations (Bézard et al. 1998; Moses et al. 2000). Figure 7 clearly shows that the disagreement between 

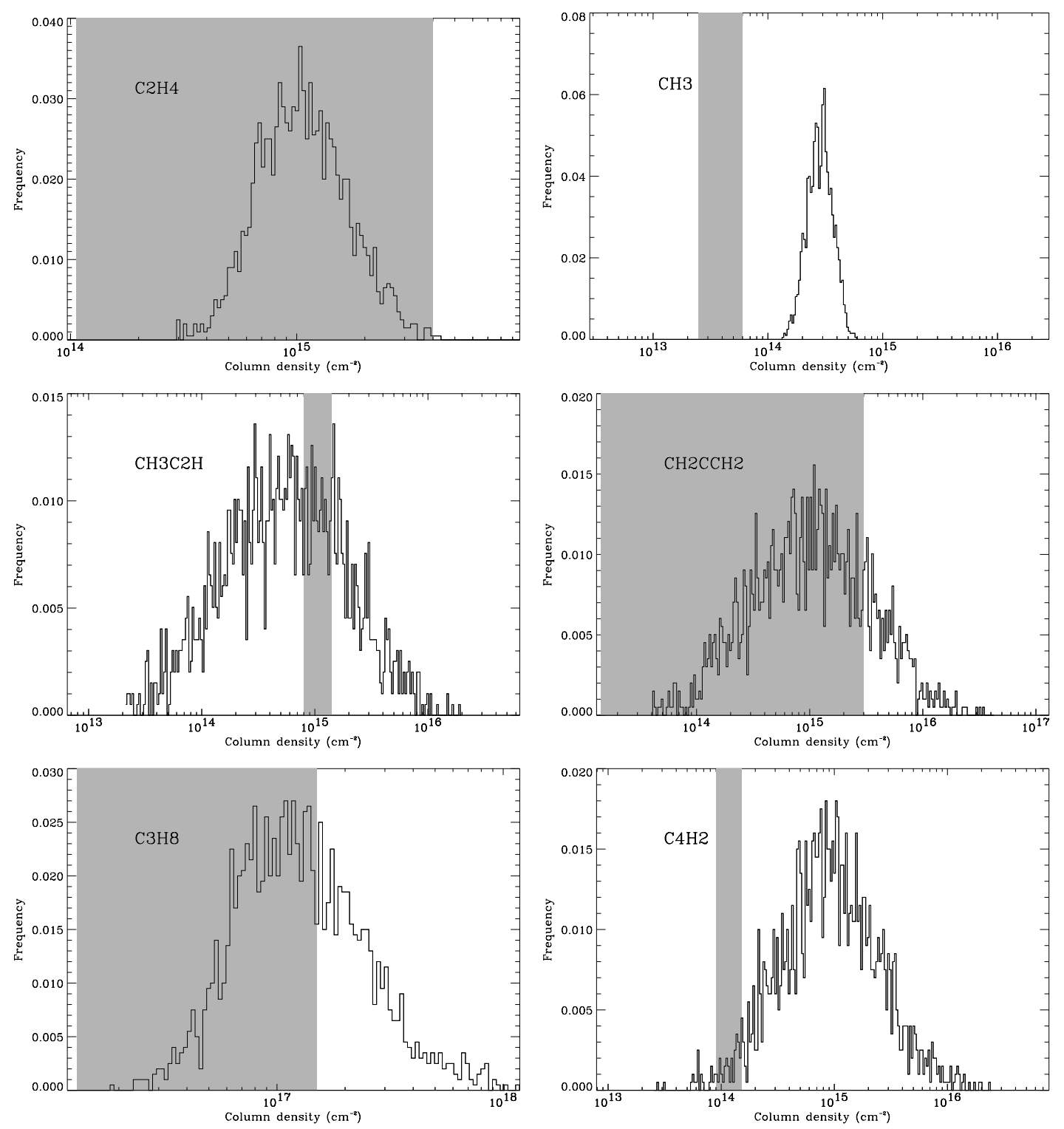

Fig. 7. Distributions of the column density of ethylene $\left(\mathrm{C}_{2} \mathrm{H}_{4}\right)$, methyl radical $\left(\mathrm{CH}_{3}\right)$, methylacetylene $\left(\mathrm{CH}_{3} \mathrm{C}_{2} \mathrm{H}\right)$, allene $\left(\mathrm{CH}_{2} \mathrm{CCH}\right)$, butane $\left(\mathrm{C}_{3} \mathrm{H}_{8}\right)$ and diacetylene $\left(\mathrm{C}_{4} \mathrm{H}_{2}\right)$. The column densities are determined for the atmospheric levels above $10 \mathrm{mbar}$ (pressure $<10 \mathrm{mbar}$ ). The dark domains correspond to the column density (above $10 \mathrm{mbar}$ ) determined by Moses et al. (2000), from ISO observations, for the respective compounds. Column densities of $\mathrm{C}_{2} \mathrm{H}_{4}, \mathrm{CH}_{2} \mathrm{CCH}_{2}$ and $\mathrm{C}_{3} \mathrm{H}_{8}$ are upper limits.

our results and the observations is highly significant. In the next section, we discuss the implications of this result.

From our results, it is possible to enumerate the number of runs that match all the observations together. According to Moses et al. (2000), ISO data analysis produced mole fraction of $\mathrm{C}_{2} \mathrm{H}_{6}, \mathrm{C}_{2} \mathrm{H}_{2}, \mathrm{CH}_{3} \mathrm{C}_{2} \mathrm{H}, \mathrm{CH}_{3}$ and $\mathrm{C}_{4} \mathrm{H}_{2}$ and the upper limits of $\mathrm{C}_{2} \mathrm{H}_{4}, \mathrm{CH}_{2} \mathrm{CCH}_{2}, \mathrm{C}_{3} \mathrm{H}_{8}$ and $\mathrm{C}_{6} \mathrm{H}_{2}$. The mole fractions of $\mathrm{CH}_{4}$ used to constrain the model comes from Festou \& Atreya (1982) and Smith et al. (1983). We have shown that the agreement between our model and the different observations derived from ISO is quite good except for $\mathrm{CH}_{3}$. We found 433 runs $(22 \%)$ that give altitude profiles that match $\mathrm{CH}_{4}$, $\mathrm{C}_{2} \mathrm{H}_{2}$ and $\mathrm{C}_{2} \mathrm{H}_{6} ; 29$ runs (1.5\%) that match $\mathrm{CH}_{4}, \mathrm{C}_{2} \mathrm{H}_{2}, \mathrm{C}_{2} \mathrm{H}_{6}$, $\mathrm{CH}_{3} \mathrm{C}_{2} \mathrm{H}, \mathrm{CH}_{2} \mathrm{CCH}_{2}, \mathrm{C}_{3} \mathrm{H}_{8}$ and $\mathrm{C}_{6} \mathrm{H}_{2}$ but no run that matches the same set of compounds plus $\mathrm{C}_{4} \mathrm{H}_{2}$. An inadequate chemical scheme or eddy diffusion profile may be invoked in order to explain the discrepancy for $\mathrm{C}_{4} \mathrm{H}_{2}$. More precisely, the lower $\gamma_{2}$ is (see Sect. 2.3), the more numerous the number of runs that match $\mathrm{C}_{4} \mathrm{H}_{2}$ observations $\left(9.2 \%\right.$ for $\gamma_{2}=0.75,3.2 \%$ for $\gamma_{2}=0.9,1.4 \%$ for $\gamma_{2}=1.05$; these results have been obtained after 500 runs for each value of $\gamma_{2}$ ). Note that uncertainties of observations as a function of pressure levels are not included in the comparison. So, we underestimate the number of runs that match observations.

Therefore, we can conclude that if we take the uncertainties on the computed mole fractions into account, we have an agreement between the results of our model and the abundances derived from ISO observations (except for $\mathrm{CH}_{3}$ ), on the contrary to what have been previously stated by Ollivier et al. (2000). However, it does not mean that the chemical scheme used in 
our photochemical model is perfect. It means that, under the present assumptions, the chemical scheme is appropriate to explain the abundances of compounds detected in the stratosphere of Saturn. It also means that a few modifications in the chemical scheme (by taking different reaction rates found in the literature) or in the eddy diffusion coefficient (see discussion) could be done without changing the above conclusion.

\section{Discussion}

\subsection{Methyl radical abundance}

The methyl radical has been detected in Saturn's atmosphere from the observations of ISO by Bézard et al. (1998). They reported that the $\mathrm{CH}_{3}$ observed column density is in the range $1.5-7.5 \times 10^{13}$ molecules $\mathrm{cm}^{-2}$, well below the value predicted by the photochemical model of Moses et al. (2000). Ollivier et al. (2000) also showed that their photochemical model overestimates the $\mathrm{CH}_{3}$ column density. Recently, Moses et al. (2000) reexamined the ISO observations and found a column density equal to $4_{-1.5}^{+2} \times 10^{13}$ molecules $\mathrm{cm}^{-2}$ which is consistent with their photochemical results. This agreement has been obtained by changing the reaction rate of the methyl recombination through three body reaction (R2: $\mathrm{CH}_{3}+\mathrm{CH}_{3}+$ $\left.\mathrm{M} \rightarrow \mathrm{C}_{2} \mathrm{H}_{6}+\mathrm{M}\right)$. There are presently no measurements of the methyl recombination rate at low temperatures (representative of Saturn's stratosphere, around 140-150 K) and at low pressures. The available values come from different extrapolations of the reaction rates at higher temperatures which lead to very different estimations. Atreya et al. (1999) showed that a factor of about 300 is found between two different estimations of the reaction rate at $140 \mathrm{~K}$.

The choice of the reaction rate strongly affects the computed $\mathrm{CH}_{3}$ column density and, as shown by Moses et al. (2000), if one includes the three-body methyl recombination rate of MacPherson et al. (1983), which is much faster, then the $\mathrm{CH}_{3}$ column density is in agreement with ISO observations. Ollivier et al. (2000) also discussed this point. In the present study, the methyl recombination rate is similar to the one used in Ollivier et al. (2000), and we confirm the inability to reproduce the observed methyl column density. The present work offers additional and essential information: the observed column density is well below the uncertainties of the computed abundance and column density. Although we give only a lower limit to the uncertainties in model profiles and column densities, it is clear now that the chemistry of $\mathrm{CH}_{3}$ is not well understood. Many solutions exist that could lead to a better agreement: as noticed in Moses et al. (2000), we could also consider a higher rate for the reaction between $\mathrm{CH}_{3}$ and $\mathrm{H}$ which produces methane through a three-body reaction. This reaction is actually one of the major loss mechanisms for $\mathrm{CH}_{3}$ and a higher rate would lead to a lower methyl abundance. From a comparison between the photochemical model of Saturn and Neptune, Ollivier (2000) demonstrates the inability of this mechanism to be a good solution. However, this latter work should be reanalyzed including the effect of uncertainties on reaction rates. After all, the solution adopted by Moses et al. (2000) is presently favoured and experiments have to be conducted to evaluate the rate of the reaction (R2) at low temperatures.

\subsection{The eddy diffusion coefficient}

Practically, the eddy diffusion coefficient profile can be obtained from the adjustement of the computed mole fractions to the observed values. More precisely, if the chemical scheme is correct (i.e. the main reactions are taken into account and all the rates are known) and if there is no unknown physical nor chemical process in the atmosphere (i.e. a process not included in the model), one expects to find the best profile with this method. According to the results presented here, we think that, even in the best of cases, it may not be so easy to constrain the eddy diffusion coefficient, since many profiles may give computed mole fractions in agreement with the whole set of observations. Actually, the existence of uncertainties on the calculated mole fractions produces an uncertainty on the eddy diffusion coefficient profile. Its evaluation is a very important task, but the amount of computer time needed to estimate it is very significant and a forthcoming paper will deal with this particular subject. However, in order to illustrate this point, we have made a few tests on the sensitivity of computed mole fractions to the eddy diffusion coefficient. For instance, we have multiplied our nominal eddy diffusion profile by a factor of 0.5 for pressure levels greater than $10^{-3}$ mbar. In over 500 runs, 6 runs (1.2\%) match $\mathrm{CH}_{4}, \mathrm{C}_{2} \mathrm{H}_{2}, \mathrm{C}_{2} \mathrm{H}_{6}, \mathrm{CH}_{3} \mathrm{C}_{2} \mathrm{H}, \mathrm{CH}_{2} \mathrm{CCH}_{2}$, $\mathrm{C}_{3} \mathrm{H}_{8}$ and $\mathrm{C}_{6} \mathrm{H}_{2}$ (about as much as the nominal model). We have also changed the slope of the profile with different values of $\gamma_{2}$. The number of runs that match $\mathrm{CH}_{4}, \mathrm{C}_{2} \mathrm{H}_{2}, \mathrm{C}_{2} \mathrm{H}_{6}$, $\mathrm{CH}_{3} \mathrm{C}_{2} \mathrm{H}, \mathrm{CH}_{2} \mathrm{CCH}_{2}, \mathrm{C}_{3} \mathrm{H}_{8}$ and $\mathrm{C}_{6} \mathrm{H}_{2}$ are $0.4 \%$ for $\gamma_{2}=0.75$, $4.2 \%$ for $\gamma_{2}=0.9,2.8 \%$ for $\gamma_{2}=1.2$ and $0.0 \%$ for $\gamma_{2}=1.3$.

\section{Conclusion}

We have developed a Monte-Carlo code to take photolysis and reaction rate uncertainties into account in a 1-D photochemical model. This model has been applied to the photochemistry of hydrocarbons in the stratosphere of Saturn. Results show that the uncertainties on computed mole fraction profiles are significant. For most of hydrocarbons, the uncertainties on computed abundances are much larger than the estimated uncertainties on abundances inferred from observations. In particular, ethane and acetylene, the most abundant hydrocarbons in Saturn's atmosphere after methane, are affected by an uncertainty factor of about 1.5 , and the mole fractions of complex hydrocarbons, which are the products of a more complex chemistry, are affected by a large uncertainty factor, ranging from 2 to 24 .

We have shown that, despite the uncertainties on abundances, the model is not able to explain the low abundance of $\mathrm{CH}_{3}$ inferred from ISO. As mentioned by Moses et al. (2000), it is important to evaluate the rate of the methyl recombination at low temperature $\left(\mathrm{CH}_{3}+\mathrm{CH}_{3}+\mathrm{M} \rightarrow \mathrm{C}_{2} \mathrm{H}_{6}+\mathrm{M}\right)$.

By comparison with ISO observations, we have shown that our chemical scheme is satisfactory, with some reluctance for $\mathrm{C}_{4} \mathrm{H}_{2}$. Further studies on the sensitivity of $\mathrm{C}_{4} \mathrm{H}_{2}$ abundance to the eddy diffusion coefficient are needed. Since Moses et al. (2000) found a chemical scheme that give a computed 
abundance in agreement with observations, a comprehensive comparison of the chemical schemes should be done.

Photochemical models are useful to constrain the physical and chemical parameters of planetary atmospheres. In particular, they can give constraints on molecular abundances, eddy diffusion coefficient, boundary conditions (e.g. influx of matter), chemical schemes, etc. We have shown that it is essential to include the uncertainties of photolysis and reaction rates in photochemical models. The main implication is that uncertainties on computed abundances might not constrain chemical and physical parameters as tightly as expected. We have seen that altitude profiles of molecular abundances may vary drastically from one run to another. The modification of the chemical scheme (addition of a reaction or modification of a reaction rate) might give no conclusive results since observed abundances may still be in agreement with the large uncertainties of computed profiles. Several eddy diffusion profiles may give satisfactory agreement with observations without changing the chemical scheme.

Extensive experimental studies are necessary to improve our knowledge of hydrocarbon reaction rates, especially at low temperature. It is important: (1) to identify the main reactions (using photochemical models or other techniques such as the one developed by Dobrijevic et al. 1995), (2) to evaluate precisely the uncertainty factors of these rates and (3) to lower these uncertainties at low temperatures. This improvement is a prerequisite for a more efficient use of photochemical modeling.

\section{Future works}

The present work is the first step of forthcoming works, among which:

- As stated previously, the best way to compare models and observations (infrared spectra for instance) is to use results of photochemical models (altitude profiles of mole fractions) and a radiative transfer code to create synthetic spectra. So, the next step will be to evaluate the effect of uncertainties of chemical rates on synthetic spectra.

- A comprehensive study of the sensitivity of abundance distributions to the eddy diffusion coefficient should be done. This work might give the degree of uncertainty on this coefficient.

- This work can be used to study other atmospheric properties like: abundance of tropospheric compounds, influx of external molecules by interplanetary dust, etc.

- A comprehensive cross-examination of extensive databases has to be done in order to evaluate the reaction rate uncertainties.
Acknowledgements. J. L. Ollivier wishes to thank J. I. Moses for helpful discussions on the chemical scheme

\section{References}

Atkinson, D. L., Baulch, D. L., Hampson, R. F., et al. 1992, J. Phys. Chem. Ref. Data, 21, Suppl. IV, 1125

Atreya, S. K., Edgington, S. G., Encrenaz, T., \& Feuchtgruber, H. 1999, The Universe as seen by ISO, ESA-SP427

Baulch, D. L., Cobos, C. J., Cox, R. A., et al. 1992, J. Chem. Phys. Ref. Data, 21, 411

Bézard, B., Feuchtgruber, H., Moses, J. I., \& Encrenaz, T. 1998, A\&A, 334, L41

Canosa, A., Sims, I. R., Travers, D., Smith, I. W. M., \& Rowe, B. R. 1997, A\&A, 323, 644

Conrath, B. J., Gautier, D., Hanel, R. A., \& Hornstein, J. S. 1984, ApJ, 282,807

Conrath, B. J., \& Gautier, D. 2000, Icarus, 144, 124

Courtin, R., Gautier, D., Marten, A., Bézard, B., \& Hanel, R. 1984, ApJ, 287, 899

De Graauw, Th., Feuchtgruber, H., Bézard, B., et al. 1997, A\&A, 321, L13

DeMore, W. B., Sander, S. P., Golden, D. M., et al. 1994, JPL Publ., 94

Dobrijevic, M., Parisot, J. P., \& Dutour, I. 1995, Planet. Space Sci., 43,15

Dobrijevic, M. 1996, Ph.D. Thesis, Université de Bordeaux 1.

Dobrijevic, M., \& Parisot, J. P. 1998, Planet. Space Sci., 46, 491

Festou, M. C., \& Atreya, S. K. 1982, Geophys. Res. Lett., 9, 1147

Laufer, A. H., Gardner, E. P., Kwok, T. L., \& Yung, Y. L. 1983, Icarus, 56, 560

MacPherson, M. T., Pilling, M. J., \& Smith, M. J. C. 1983, Chem. Phys. Lett., 94, 430

Mordaunt, D. H., Lambert, I. R., Morley, G. P., et al. 1993, J. Chem. Phys., 98, 2045

Moses, J. I., Bézard, B., Lellouch, E., et al. 2000, Icarus, 143, 244

Ollivier, J. L. 2000, Ph.D. Thesis, Université de Bordeaux 1.

Ollivier, J. L., Dobrijevic, M., \& Parisot, J. P. 2000, Planet. Space Sci., 48, 699

Sims, I. R., Queffelec, J-L., Travers, D., et al. 1993, Chem. phys. lett., 211, 461

Smith, G. R., Shemansky, D. E., Holberg, J. B., et al. 1983, J. Geophys. Res., 88, 8667

Smith, N. S., \& Raulin, F. 1998, BAAS, 30

Stewart, R. W., \& Thompson, A. M. 1996., J. Geophys. Res., 101, 20953

Stolarski, R. S., Butler, D. M., \& Rundel, R. D. 1978, J. Geophys. Res., 83, 3074

Thompson, A. M., \& Stewart, R. W. 1991, J. Geophys. Res., 96, 13089

Wilson, E. H., \& Atreya, S. K. 2000, J. Geophys. Res., 105, 20263 